\title{
Epstein-Barr Virus-Induced Mononucleosis as an Imitator of Severe Preeclampsia
}

\author{
S. Allison Staley, MD, MPH ${ }^{1}$ Marcela C. Smid, MD, MS, MA ${ }^{2}$ Sarah K. Dotters-Katz, MD² \\ Elizabeth M. Stringer, MD, MSCE
}

${ }^{1}$ Department of Obstetrics and Gynecology, University of North Carolina, Chapel Hill, North Carolina

2 Division of Maternal-Fetal Medicine, Department of Obstetrics and Gynecology, University of North Carolina, Chapel Hill, North Carolina

Address for correspondence S. Allison Staley, MD, MPH, 3010 Old Clinic Building CB \#7516, Chapel Hill, NC 27599-7516

(e-mail: astaley@unch.unc.edu).

Am J Perinatol Rep 2016;7:e5-e7.

\begin{abstract}
Keywords

- preeclampsia

- mononucleosis

- preterm birth

- maternal

- thrombocytopenia

Background In pregnancy, conditions presenting with hematologic abnormalities, transaminitis, and proteinuria pose diagnostic challenges in pregnancy.

Case We present the case of an 18-year-old woman, G1P0, at 33 weeks' gestation with fever of unknown cause, who developed progressively elevated liver enzymes, proteinuria, and thrombocytopenia, due to Epstein-Barr virus (EBV) infection.

Conclusion Acute infection with EBV should be included in the differential diagnosis of preeclampsia with severe features, particularly in the setting of fever. Supportive treatment and observation may prevent iatrogenic preterm birth.
\end{abstract}

Conditions presenting with hematologic abnormalities, elevated liver enzymes, and proteinuria in pregnancy may pose a diagnostic challenge. Some of the more commonly described disorders that may present with these findings include preeclampsia with severe features/HELLP syndrome, acute fatty liver of pregnancy (AFLP), thrombotic thrombocytopenic purpura (TTP), hemolytic uremic syndrome (HUS), and acute exacerbation of systemic lupus erythematosus. ${ }^{1}$ Accurate diagnosis is imperative because subsequent management and treatment vary and may result in significant neonatal and maternal consequences. For example, based on the diagnosis, preterm delivery may be indicated. We present a case of Epstein-Barr virus (EBV) infection in pregnancy mimicking many of the same signs and symptoms of preeclampsia with severe features.

\section{Case}

An 18-year-old woman, G1P0, presented at $33^{2 / 7}$ weeks estimated gestational age with an uncomplicated singleton pregnancy, with fever of unknown cause. She reported fever for 2 days with associated headache and right upper quadrant abdominal pain. She initially presented 24 hours after onset of symptoms to a local urgent care clinic, where she was then referred to a local hospital for evaluation. Prior to transfer to our institution, she was reported to have a normal white blood cell (WBC) count, elevated liver enzymes, and thrombocytopenia. A right upper quadrant ultrasound was reported as normal as well as a chest radiograph. She was given one dose of betamethasone for fetal lung maturity, started on prophylactic magnesium infusion given concern for possible HELLP syndrome, and transferred to our institution for further evaluation and management.

On presentation, she was febrile $\left(38.6^{\circ} \mathrm{C}\right)$ with tachycardia, but normotensive with blood pressure 109 to $126 / 67$ to 81 . She appeared fatigued, but in no acute distress. All components of her physical exam were normal except for findings of elevated jugular venous distention and tachycardia with a holosystolic murmur. On review of systems, she denied upper respiratory symptoms, cough, sore throat, lymphadenopathy, dysuria, abnormal vaginal discharge, or recent sick contacts or known exposures.

Initial fetal nonstress test was reactive with fetal heart rate elevated at 160 beats per minute. A bedside ultrasound was performed and her fetus was found to be in vertex position with maximum vertical pocket of $1.9 \mathrm{~cm}$, estimated fetal weight of $2,144 \mathrm{~g}$ (44th percentile), and biophysical profile of 8 out of 10 . received

August 15, 2016

accepted after revision

October 20, 2016
DOI http://dx.doi.org/

10.1055/s-0036-1597265. ISSN 2157-6998.
Copyright $\odot 2017$ by Thieme Medical Publishers, Inc., 333 Seventh Avenue, New York, NY 10001, USA. Tel: +1(212) 584-4662.

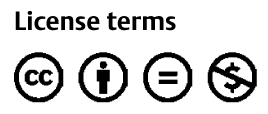


She denied any leakage of fluid or abnormal vaginal discharge. A sterile speculum was performed, which demonstrated physiologic discharge and a visually closed cervix. Sampled discharge was negative for nitrazine and ferning. AmniSure (Germantown, Maryland) testing was negative.

Initially, the differential diagnosis was broad, including HELLP syndrome, AFLP, acute viral hepatitis, TTP, HUS, cholecystitis, chorioamnionitis, pneumonia, pyelonephritis, meningitis, endocarditis, autoimmune disease, and other infectious etiologies, including human immunodeficiency virus, Rocky Mountain spotted fever (RMSF), infectious mononucleosis, influenza, cytomegalovirus (CMV), respiratory syncytial virus (RSV), parainfluenza, and disseminated herpes simplex virus (HSV). Blood and urine cultures were obtained. She was treated with broad-spectrum antibiotics as well as continued on magnesium protocol for seizure prophylaxis. She was also treated supportively with intravenous fluids and acetaminophen for fever. In addition, pad counts were performed to assess for leakage of amniotic fluid. She remained on labor and delivery for frequent vital signs, continuous fetal monitoring, as well as serial laboratory assessments to trend her blood count, liver enzymes, and lactate dehydrogenase (LDH). Continuous monitoring remained Category I. At the conclusion of hospital day 1, the following laboratory values were available: WBC count $4.7\left(\times 10^{9} / \mathrm{L}\right)$, hematocrit $31.5 \%$, platelets (PLT) $99\left(\times 10^{9} / \mathrm{L}\right)$, aspartate aminotransferase (AST) 114 (U/L), alanine aminotransferase (ALT) 185 (U/L), LDH 764 (U/L), C3 complement 155 (mg/dL), C4 complement 52.1 (mg/dL), rapid plasma reagin negative, CMV immunoglobulin (Ig)M negative, HSV1 IgG negative, HSV2 IgG negative, HSV IgM negative, hepatitis $\mathrm{A} \operatorname{IgG}$ nonreactive, urine protein-to-creatinine ratio 1.33, and urinalysis was significant for moderate leukocyte esterase and positive protein. At this time, the differential diagnosis was preeclampsia with severe features/HELLP syndrome, TTP, or infectious etiology.

On hospital day 2 , the patient remained stable, but recurrently febrile with temperature to $39.6^{\circ} \mathrm{C}$. Her management was continued as previously described. Continuous fetal monitoring remained Category I. Blood pressures ranged 107 to $124 / 73$ to 80 . Given findings of oligohydramnios on bedside ultrasound, a formal ultrasound was performed. Findings demonstrated a singleton fetus with estimated fetal weight 2,462 $\mathrm{g}$ (47th percentile), amniotic fluid index $7.7 \mathrm{~cm}$, and normal umbilical artery Doppler. During hospital day 2 , on serial laboratory evaluation, the following values were notable: WBC $2.3\left(\times 10^{9} / \mathrm{L}\right)$, AST elevated to $385(\mathrm{U} / \mathrm{L})$, ALT 198 (U/L), LDH 2,210 (U/L), and PLT $38\left(\times 10^{9} / \mathrm{L}\right)$. On hospital day 2 , the following results were available: urine drug screen negative and ADAMTS13 78\% (low). To rule out TTP, peripheral smear was obtained, which demonstrated lymphopenia with atypical lymphocytes. In addition, viral evaluation yielded negative results for the following: RMSF antibody, influenza $A$, influenza $B$, adenovirus, metapneumovirus, parainfluenza viruses 1 to 4, RSV, and rhinovirus. A mononuclear spot test was performed, which yielded a positive result. EBV IgM was positive, and EBV IgG was negative. EBV quantitative was 6,606 copies/mL.
Based on these results, infectious disease and hematology services were consulted, and an abdominal ultrasound was obtained to evaluate the spleen. Complete abdominal ultrasound demonstrated normal gallbladder and kidneys, but an enlarged spleen measuring $16.3 \mathrm{~cm}$ in length. On hospital day 2 , the patient was seen by the infectious disease consulting service, whose team was in agreement with current management and ongoing antibiotic therapy until cultures returned negative. Because she demonstrated normal BPs, normal fetal growth and testing, and was definitely diagnosed with EBVinduced mononucleosis, suspicion for preeclampsia was low. Magnesium protocol was discontinued.

On hospital day 3, initial blood and urine cultures at the referring hospital returned negative. Antibiotic therapy was discontinued. The patient remained intermittently febrile, but physical exam remained otherwise negative. Fetal testing also remained reassuring with continuous fetal monitoring. She was seen by hematology and infectious disease. Following consultation, continued expectant management with fever control, serial laboratories, and daily fetal monitoring was recommended. Per hematology recommendation, the patient was given PLT transfusion as needed to maintain a level of 50 or greater, pending possible preterm labor or need for delivery.

Because the initial urine protein-to-creatinine ratio was 1.33 on admission, a 24-hour urine protein was collected, which showed $2,146 \mathrm{mg}$ on hospital day 3 . Urine sediment only demonstrated squamous cells. Creatinine remained stable and normal throughout her admission. Comparison of urine dips from her prenatal care provider demonstrated previously negative urine protein. Nephrology was consulted, given this new proteinuria in setting of EBV-induced mononucleosis. Other etiologies of this new proteinuria included preeclampsia as well as high fever. As such, proteinuria was not assumed to be secondary to EBV infection alone, and the patient was monitored for elevated blood pressures and symptoms of preeclampsia. Throughout her admission, blood pressures and creatinine remained normal.

By hospital day 6 , the patient remained afebrile for greater than 24 hours without antipyretics. Simultaneously, thrombocytopenia, liver enzymes, and WBC count began to normalize on hospital day 6 . She was discharged on hospital day 8 with the following notable laboratory values: WBC $4.8\left(\times 10^{9} / \mathrm{L}\right)$, PLT $88\left(\times 10^{9} / \mathrm{L}\right)$, AST $103(\mathrm{U} / \mathrm{L})$, and ALT $128(\mathrm{U} / \mathrm{L})$.

She presented the following day at $34^{2 / 7}$ weeks gestational age with new preterm contractions. On cervical exam, she was dilated $2 \mathrm{~cm}$ and $90 \%$ effaced at -2 station in cephalic presentation. The patient was monitored expectantly, and she precipitously delivered vaginally on the day of admission. Of note, on presentation, repeat laboratory evaluation demonstrated the following: WBC $10.1\left(\times 10^{9} / \mathrm{L}\right)$, PLT $139\left(\times 10^{9} / \mathrm{L}\right)$, AST $57(\mathrm{U} / \mathrm{L})$, ALT $103(\mathrm{U} / \mathrm{L})$, creatinine $0.69 \mathrm{mg} / \mathrm{dL}$, and urine protein-to-creatinine ratio 0.56 . The patient was meeting all postpartum goals on hospital day 3. She was seen by lactation services for breastfeeding support. She was exclusively breastfeeding and pumping, and her infant was stable in the neonatal intensive care unit at the time of discharge. 


\section{Discussion}

EBV (human herpesvirus 4), more commonly known as mononucleosis, is one of the most common human viruses. The herpetic family of viruses has the ability to remain latent in the body and become reactivated. Infectious mononucleosis often begins insidiously, with vague malaise, followed by fever, sore throat, swollen posterior cervical lymph nodes, and fatigue. Some patients experience an abrupt influenza-like onset, with fever, chills, body aches, and sore throat. ${ }^{2}$ Our case highlights that EBVinduced mononucleosis may imitate preeclampsia and, in rare cases, may cause new proteinuria or renal disease. Based on prior case reports, EBV can cause collapsing focal segmental glomerulosclerosis or minimal change disease as well as acute renal failure secondary to tubulointerstitial nephritis, although these cases are rare. ${ }^{3,4}$ Furthermore, we propose that a thorough differential diagnosis be explored, including viral evaluation, particularly in setting of concomitant fever to avoid iatrogenic preterm birth due to presumed preeclampsia.

Very limited information exists on acute EBV-induced mononucleosis on pregnancy outcomes and evidence-based management in these scenarios is nonexistent. Several studies have investigated EBV reactivation and pregnancy outcomes. Women with significant EBV reactivation have been found to have significantly shorter duration of pregnancy compared with those who did not experience reactivation. Fetal mortality ad teratogenic risk, however, have not been demonstrated. ${ }^{5}$ There are few epidemiological studies that have addressed the risk of vertical EBV transmission. Notably, in a study of 2,000 newborns, no EBV-transformed cells were found in cord blood and no serological evidence of intrauterine infection was reported. ${ }^{5,6}$ Even more uncommonly, there have been no studies solely examining the effect of acute EBV-induced mononucleosis on pregnancy outcomes and directing best practice management in these scenarios. Based on our case experience and practice management recommendations in nonpregnant women, supportive treatment is preferred with a focus on symptom management, including the use of antipyretics, analgesics, fluids, and limitation of activity. ${ }^{2}$

In the nonpregnant population, most clinicians recommend acetaminophen use and avoidance of aspirin-based antipyretics or analgesics as these have been associated with splenic hemorrhage. Fever typically subsides within a week, but longer durations can occur. ${ }^{2}$ Attention to fluid management and nutrition is imperative, especially for the febrile patient in which insensible losses are not objectively quantifiable. The clinician should also review physical activity precautions with the pregnant patient as the risk for splenic rupture still exists. ${ }^{2}$ Furthermore, postpartum risks should be reviewed. Active infection with mononucleosis is a contraindication to breastfeeding as the presence of EBV can be found in human milk. However, reports on breastfed infants infected by the virus are rare. ${ }^{7}$

Our case demonstrates the potential for EBV-induced mononucleosis to be an imitator of severe preeclampsia, which may increase the risk for iatrogenic preterm birth. Although not studied in EBV, other viral infections may increase the risk of spontaneous preterm birth. ${ }^{8}$ Although vigilance for pregnancy-induced disease is paramount, expectant management of EBV with a focus on fever control and fluid management is the key. Furthermore, postpartum recommendations should also be reviewed with exclusively breastfeeding mothers.

\section{Summary}

EBV-induced mononucleosis may imitate signs and symptoms of preeclampsia with severe features and should be considered in the differential diagnosis, particularly in setting of fever.

\section{Teaching Points}

1. EBV may imitate preeclampsia with severe features and place women at risk for spontaneous and iatrogenic preterm birth.

2. Expectant management and supportive treatment are preferred in pregnant women with mononucleosis, and this form of management may prevent iatrogenic preterm birth.

3. Active infection with EBV is a contraindication to breastfeeding, but not once the patient is postviral.

\section{Disclosure}

We have no financial disclosure.

Acknowledgment

None.

\section{References}

1 Sibai BM. Imitators of severe preeclampsia. Obstet Gynecol 2007; 109(4):956-966

2 Odumade OA, Hogquist KA, Balfour HH Jr. Progress and problems in understanding and managing primary Epstein-Barr virus infections. Clin Microbiol Rev 2011;24(1):193-209

3 Lei PS, Lowichik A, Allen W, Mauch TJ. Acute renal failure: unusual complication of Epstein-Barr virus-induced infectious mononucleosis. Clin Infect Dis 2000;31(6):1519-1524

4 Mayer HB, Wanke CA, Williams M, Crosson AW, Federman M, Hammer SM. Epstein-Barr virus-induced infectious mononucleosis complicated by acute renal failure: case report and review. Clin Infect Dis 1996;22(6):1009-1018

5 Eskild A, Bruu AL, Stray-Pedersen B, Jenum P. Epstein-Barr virus infection during pregnancy and the risk of adverse pregnancy outcome. BJOG 2005;112(12):1620-1624

6 Fleisher G, Bologonese R. Epstein-Barr virus infections in pregnancy: a prospective study. J Pediatr 1984;104(3):374-379

7 Lamounier JA, Moulin ZS, Xavier CC. Recommendations for breastfeeding during maternal infections [in Portuguese]. J Pediatr (Rio J) 2004;80(5, Suppl):S181-S188

8 Habib NA, Daltveit AK, Bergsjø P, Shao J, Oneko O, Lie RT. Maternal HIV status and pregnancy outcomes in northeastern Tanzania: a registry-based study. BJOG 2008;115(5):616-624 